\title{
Performance of the Center-of-Curvature Optical Assembly during Cryogenic Testing of the James Wehb Space Telescope
}

\author{
James Hadaway - University of Alabama in Huntsville \\ Conrad Wells, Gene Olczak, Tony Whitman, Joseph Cosentino, \\ Michael Zarella, \& Mark Connolly - Harris Corp. \\ Mark Waldman - Sigma Space Corp. \\ David Chaney - Ball Aerospace \& Technologies Corp. \\ Randal Telfer - Space Telescope Science Institute
}

SPIE Astronomical Telescopes + Instrumentation

June 10, 2018 
- The James Webb Space Telescope

- JWST Cryogenic Test Overview

- Primary Mirror Testing Overview

- The Center-of-Curvature Optical Assembly

- PM Alignment

- Global PM Alignment

- PM Segment Alignment \& Phasing

- PM Measurement Results

- PM 1g WFE

- Measured vs Predicted 1g WFE

- Estimated Og WFE

- PM ROC, Conic, \& Collecting Area

- Summary \& Conclusions 


\section{The James Wehh Space Telescope [JWST]}

Telescope

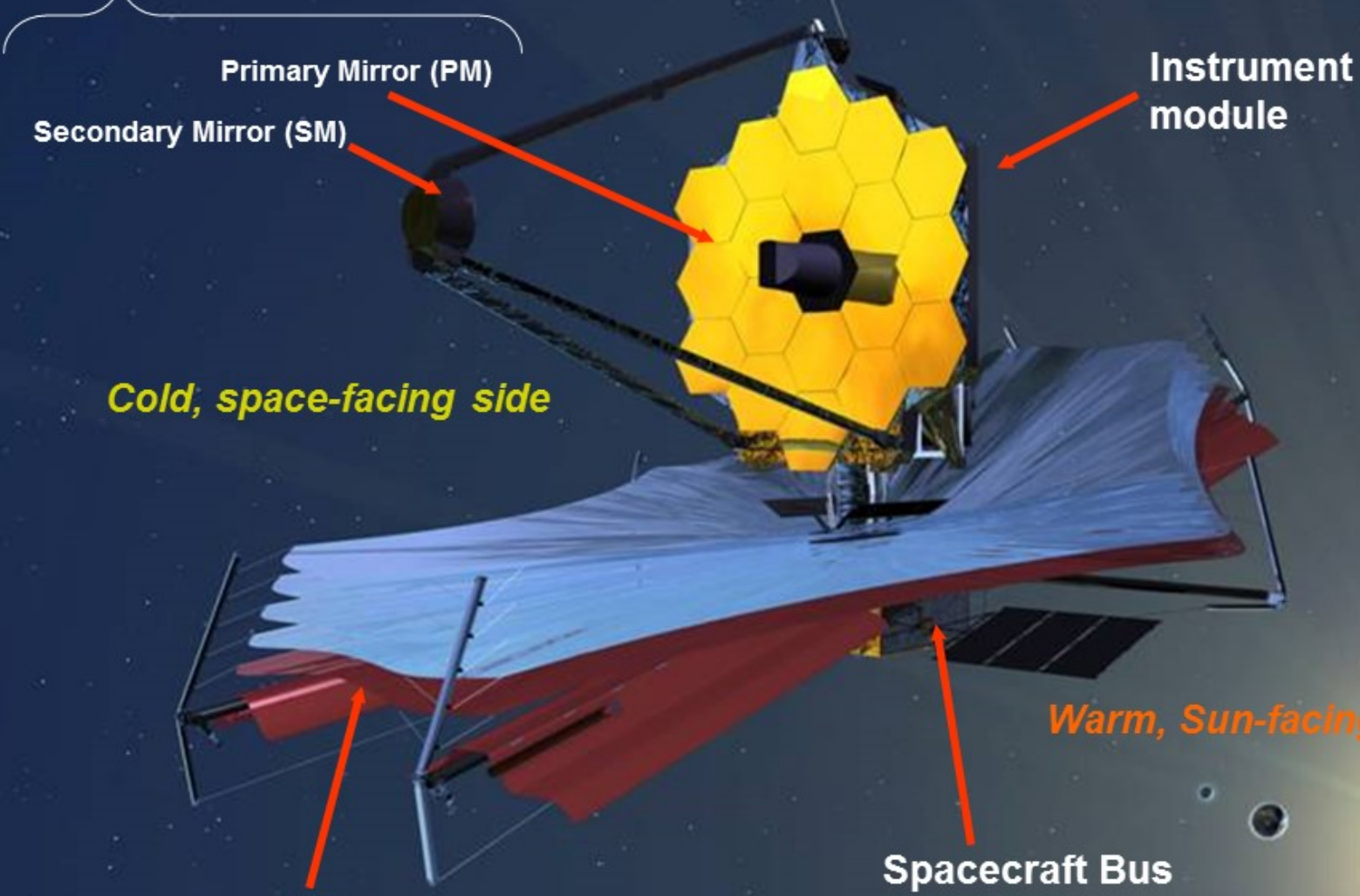

Sunshield
Instrument

- Near \& mid IR

- 3-mirror anastigmat

- Orbit around L2

- NASA, ESA, \& CSA

- 6.6 m diameter PM

- 18 segments

- Beryllium w/ gold coating

- 6-DOF \& ROC each seg

- 32-59 K operational temp 


\section{Chamber A at JSC}

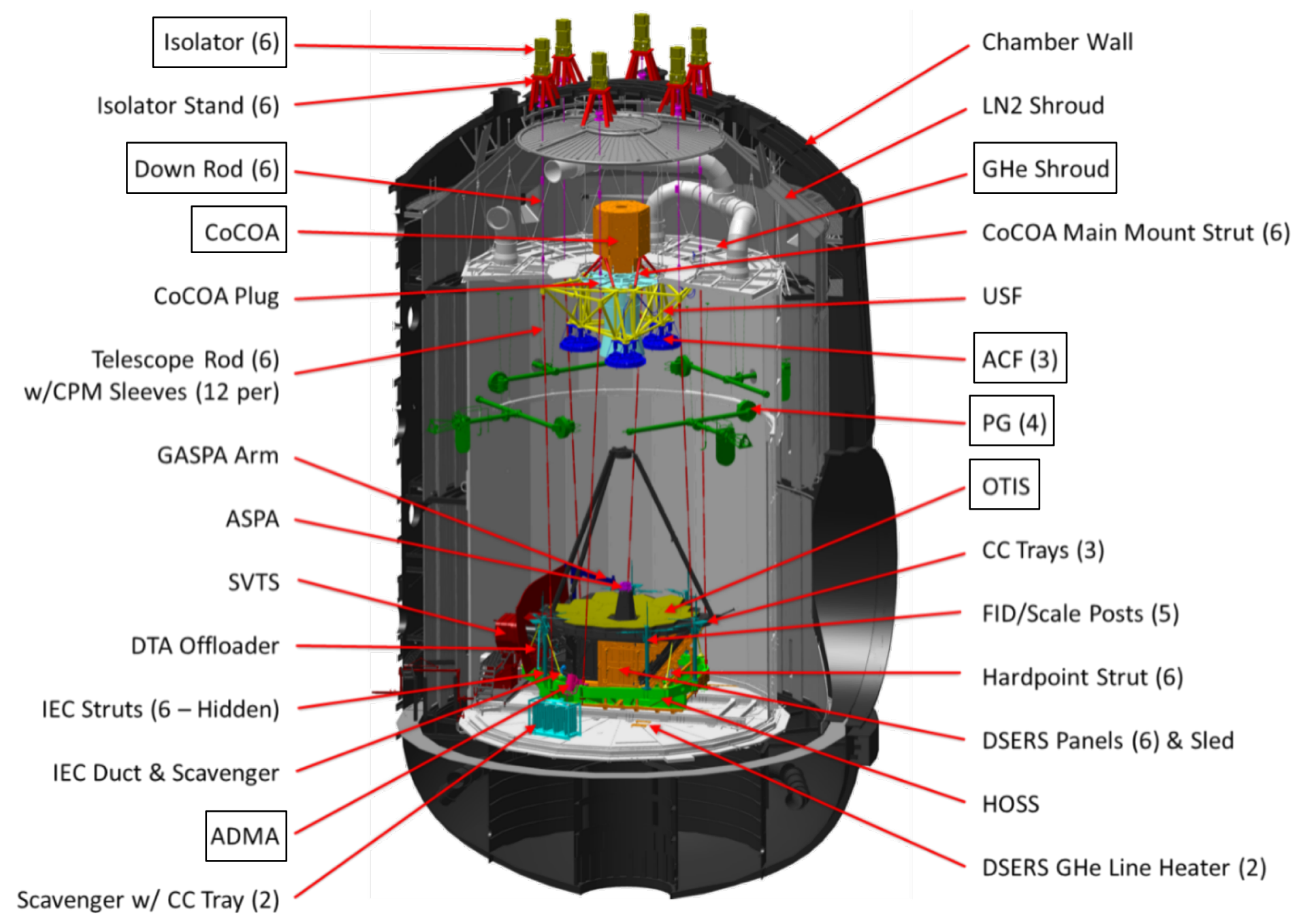




\section{IWST in Chamber A at ISC}

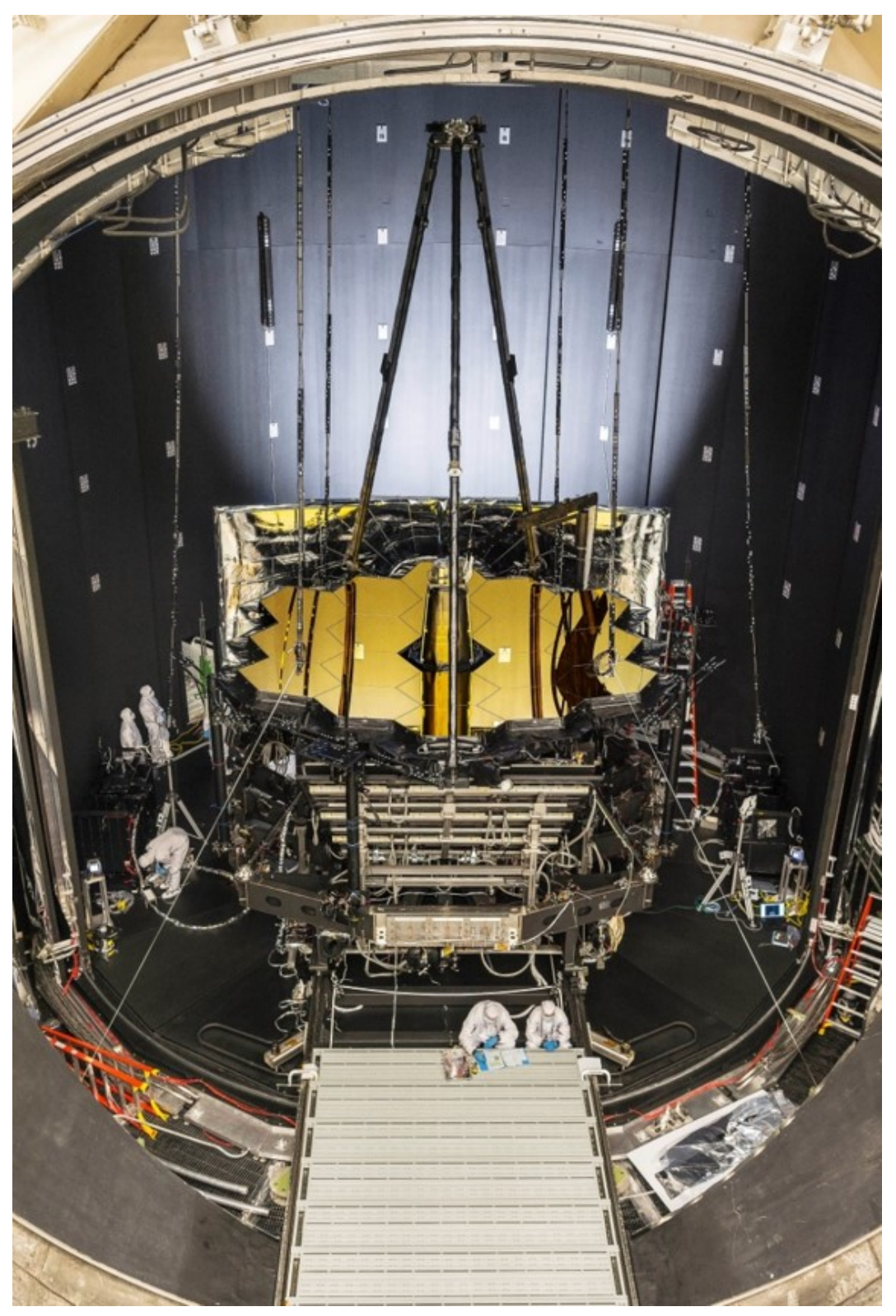




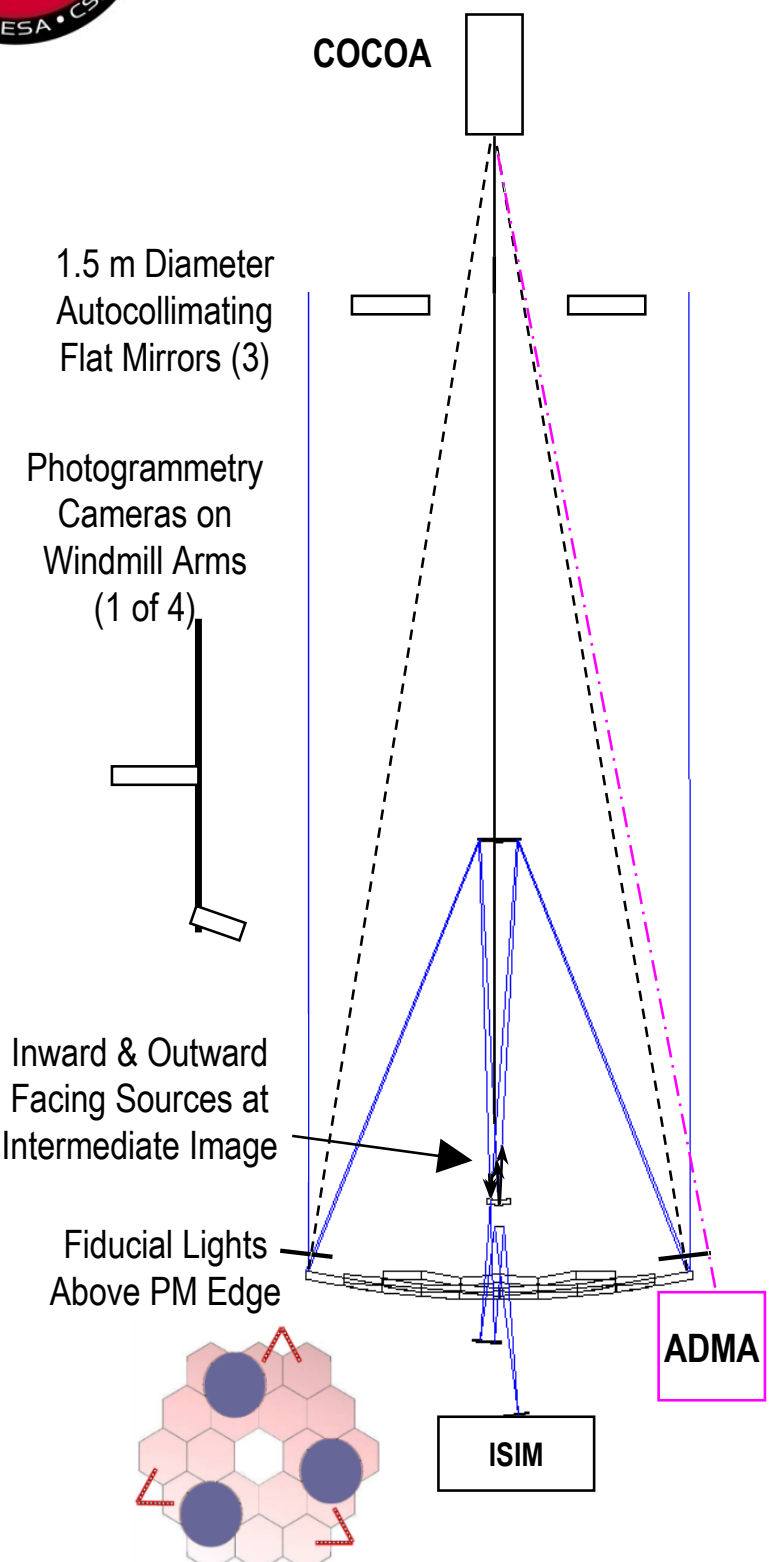

PM Alignment \& Measurement

- Photogrammetry (PG) for global positioning.

- Center-of-curvature optical assembly (COCOA) for PM alignment \& wavefront error (WFE) measurment.

- Alignment cameras for initial capture.

- Multi-wavelength interferometer (MVIF) \& reflective null for final alignment \& PM WFE measurement.

- Computer-generated-hologram (CGH) for interferometer/null WFE calibration.

- Displacement measuring interferometers (DMls) to monitor axial change during thermal distortion test.

- Fiducial lights around PM for initial alignment.

- Absolute distance meter assembly (ADMA) for axial distance/ROC. 


\section{PM Layout for JWST Gryogenic Test}

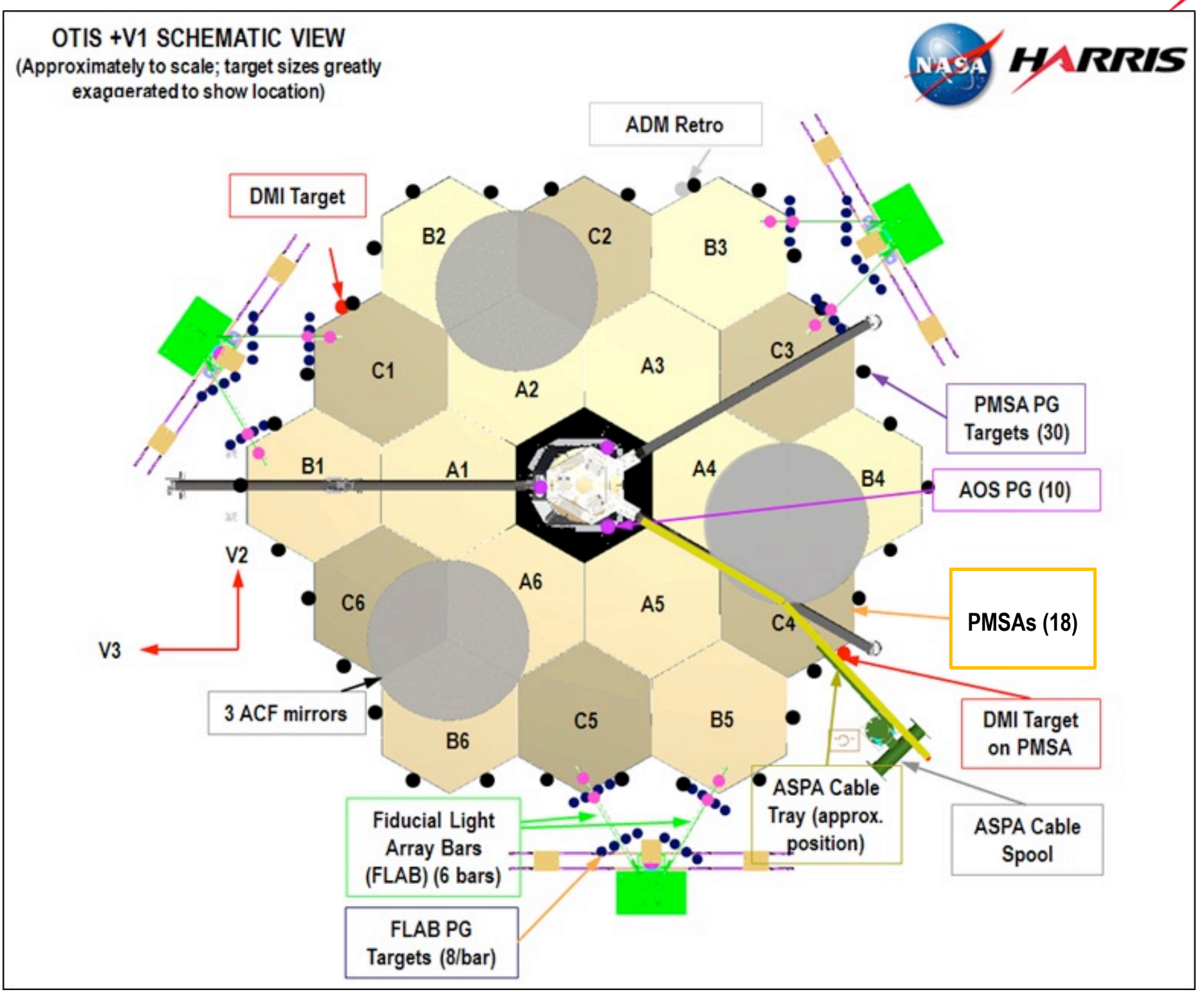


- Objectives:

- Align PM Segment Assemblies (PMSAs) into a phased PM, with proper ROC \& conic constant, and align phased PM globally to fixed Aft Optical System (AOS).

- Measure phased PM WFE, ROC, conic constant, \& collecting area in $1 \mathrm{~g}$ test environment.

- Realign \& measure WFE as required to support other testing, such as PM Thermal Distortion Figure Drift Tests and Pass-and-a-Half testing.

- Phased PM measurement results used to:

- Compare measured 1g PM WFE to prediction.

- Estimate Og PM WFE.

- Check measured 1g PM ROC \& conic constant deltas from nominal to expected uncertainties.

- Determine PM collecting area and compare to prediction. 


\section{PM Alignment Sequence}

1. Align outer PMSAs to AOS using PM.

- Only outer PMSAs capable of holding PG targets.

2. Align COCOA to outer PMSAs.

3. Align PMSAs in tilt using Coarse Alignment Subsystem.

- To within range of Fine Alignment Subsystem.

4. Align PMSAs in tilt using Fine Alignment Subsystem.

- To within range of interferometer (i.e. get fringes).

5. Align \& phase PMSAs using Multi-Wavelength Interferometer.

- Correct PMSA piston errors progressively via step-down process through incremental "synthetic wavelengths" from $15 \mathrm{~mm}$ to 17 um.

- Align PMSAs in tilt, radial decenter, clocking, \& ROC to minimize total PM WFE.

- Adjust COCOA in decenter to minimize PM tilt and pointing to minimize PM coma. Maintain axial distance with ADMA.

6. Iterate as required.
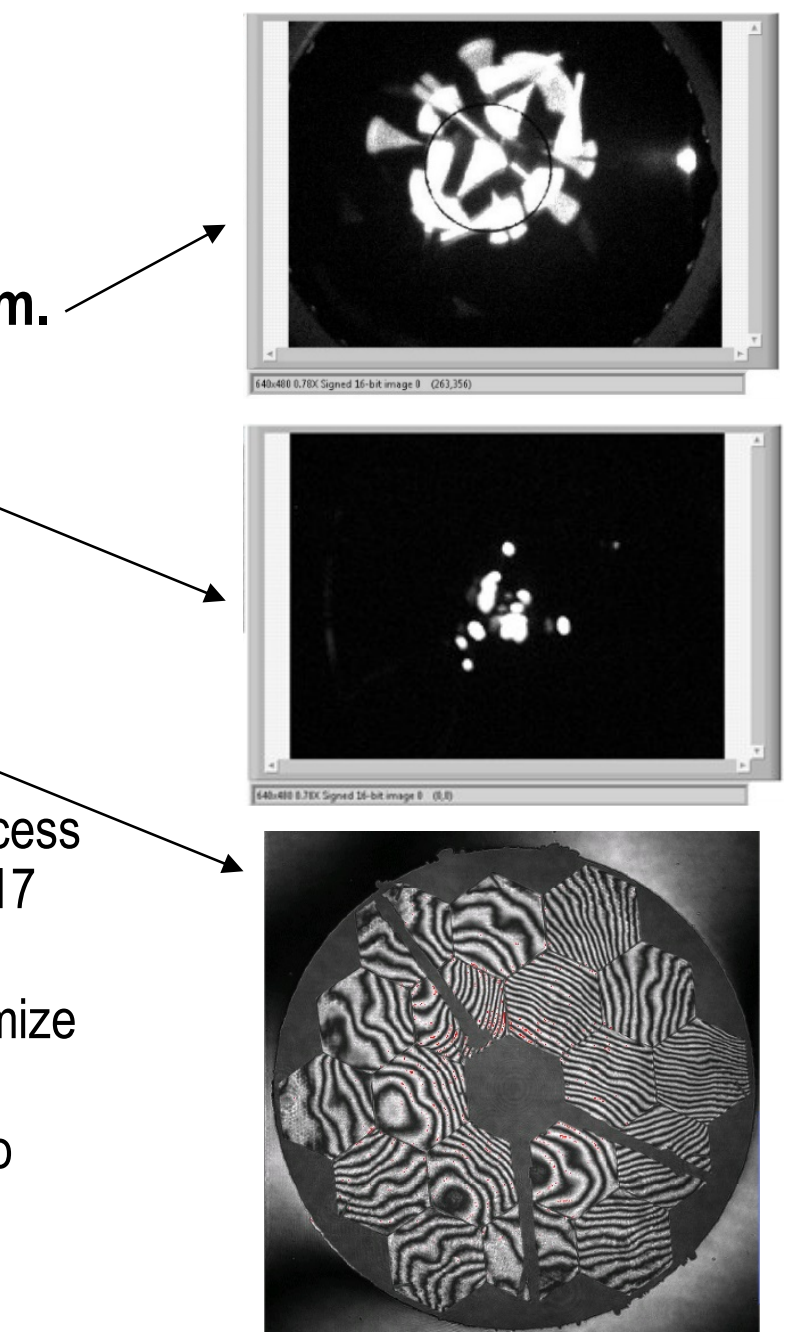
Excellent final PMSA alignment \& phasing results:

- Piston: 118 nm-PV (34 nm-RMS), meeting requirement of $\leq 150 \mathrm{~nm}-\mathrm{PV}$

- Tilt: $\leq 83$ nrad

- Decenter: $\leq 816$ um

Good PM WFE convergence:

- Clocking: $\leq 683$ urad

- Power/ROC: $\leq 10 \mathrm{~nm}-\mathrm{PV}$

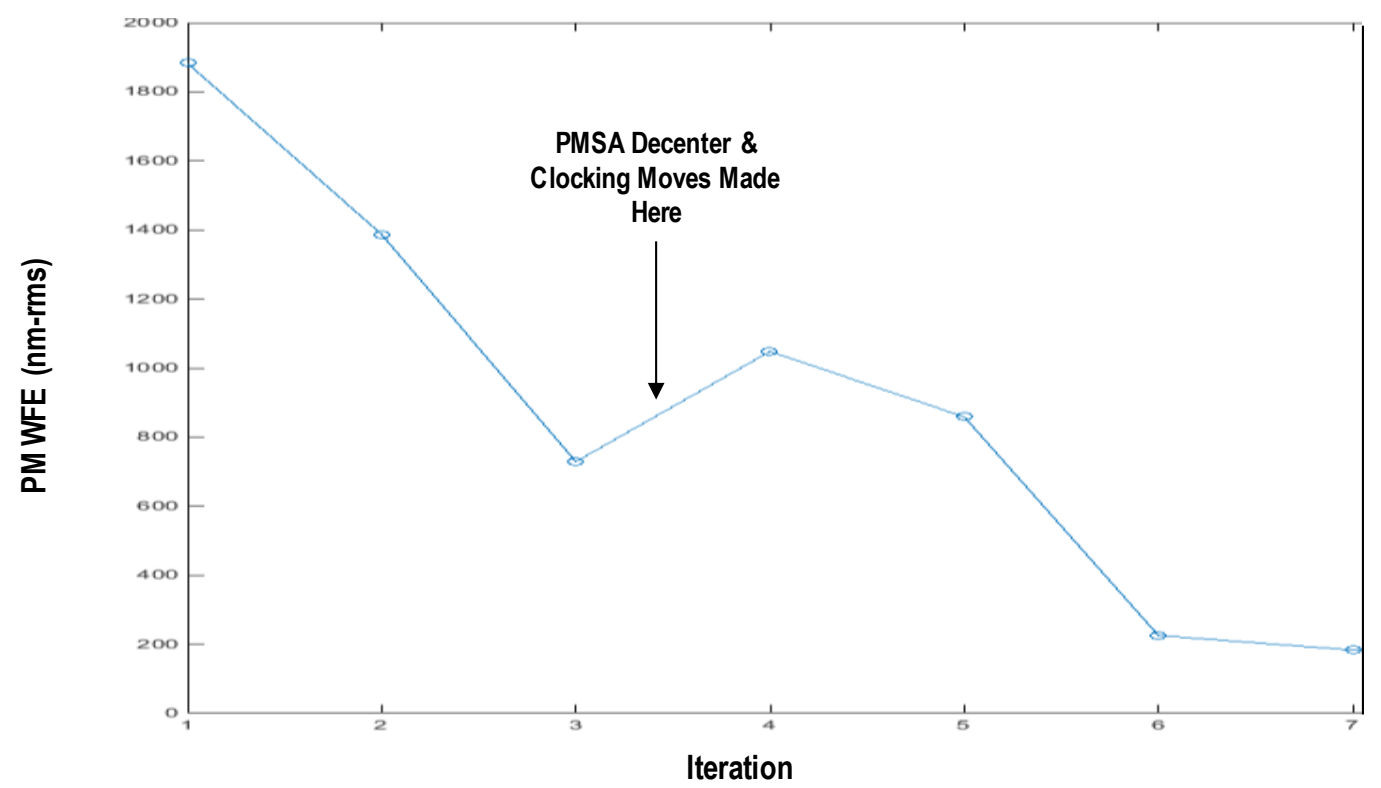




\section{PM Global Alīgnment Results}

PM global alignment results from PG after final PMSA alignment \& phasing:

\begin{tabular}{|c|c|c|}
\hline & Measured & Tolerance \\
\hline Piston $(\mathrm{mm})$ & -0.016 & \pm 0.084 \\
\hline Decenter $(\mathrm{mm})$ & 0.451 & \pm 0.030 \\
\hline Clocking $(\mathrm{mrad})$ & -0.036 & \pm 0.697 \\
\hline Tilt $(\mathrm{mrad})$ & 0.065 & \pm 0.079 \\
\hline
\end{tabular}

- During alignment of PMSAs using COCOA, PM was inadvertently misaligned in global decenter.

- Cause determined to be error in settings within code used for calculations of PMSA \& COCOA alignment moves from measured PM WFE.

- Error led to incorrect COCOA pointing, resulting in global decenter of PMSAs/PM to realign to COCOA.

- Could have corrected misalignment. But team determined that alignment was acceptable, since amount of misalignment was known.

- And ability to globally align PM to within all tolerances demonstrated during late cool-down. 

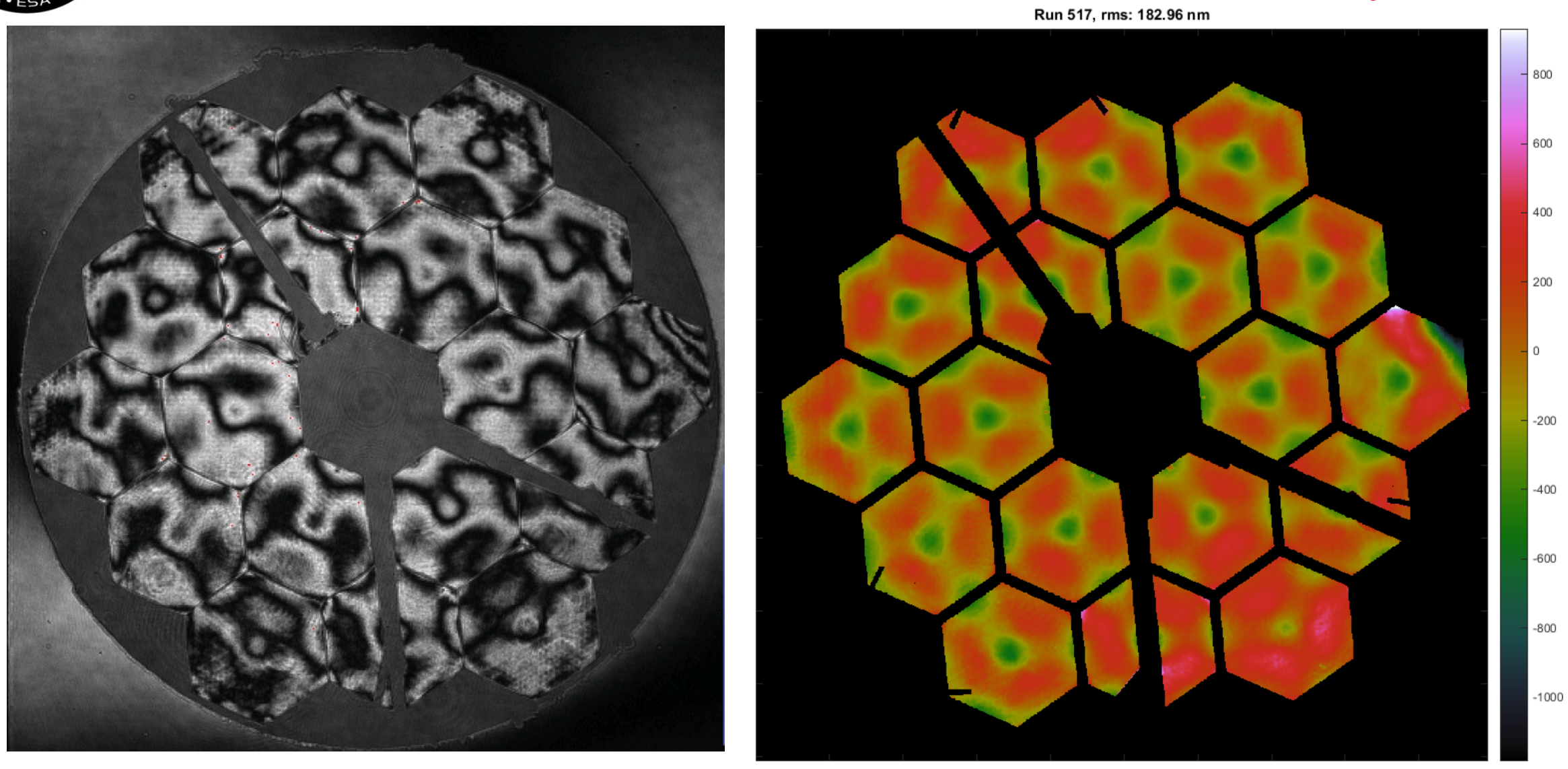

- Excellent PM WFE achieved, with low segment-level astigmatism.

- $183 \mathrm{~nm}$-rms achieved close to theoretical minimum of $158 \mathrm{~nm}$-rms.

- Unusual, uncorrectable deformation noticed on PMSA at far right.

- Determined to be from hang-up of PMSA PG target on edge closeout - not flight issue. 


\section{Final PM ROC \& Conic and Collecting Area Measurement Results}

HIRRIS

- PM ROC \& conic results both met requirements.

- See Poster 10698-136, "Setting the James Webb Space Telescope primary mirror radius-of-curvature and conic constant during cryogenic testing", by Joseph Cosentino for further details.

\begin{tabular}{|c|c|c|c|c|}
\hline Parameter & $\begin{array}{c}\text { Measured } \\
\text { Value }\end{array}$ & $\begin{array}{c}\text { Delta from } \\
\text { Nominal }\end{array}$ & $\begin{array}{c}\text { Estimated } \\
\text { Uncertainty }\end{array}$ & $\begin{array}{c}\text { Required } \\
\text { Uncertainty }\end{array}$ \\
\hline ROC $(\mathrm{mm})$ & $15,879.209$ & -0.013 & \pm 0.350 & \pm 0.400 \\
\hline Conic & -0.996692 & $-32 \mathrm{ppm}$ & $\pm 21 \mathrm{ppm}$ & $\pm 200 \mathrm{ppm}$ \\
\hline
\end{tabular}

- Predicted PM collecting area, as viewed from center-of-curvature, was $25.054 \mathrm{~m}^{2}$ with an uncertainty of $+4.3 \% /-4.2 \%$.

- Measured area of $25.411 \mathrm{~m}^{2}$ matched predicted area to $1.4 \%$, well within prediction uncertainty and below measurement uncertainty requirement of $\leq 5 \%$. 


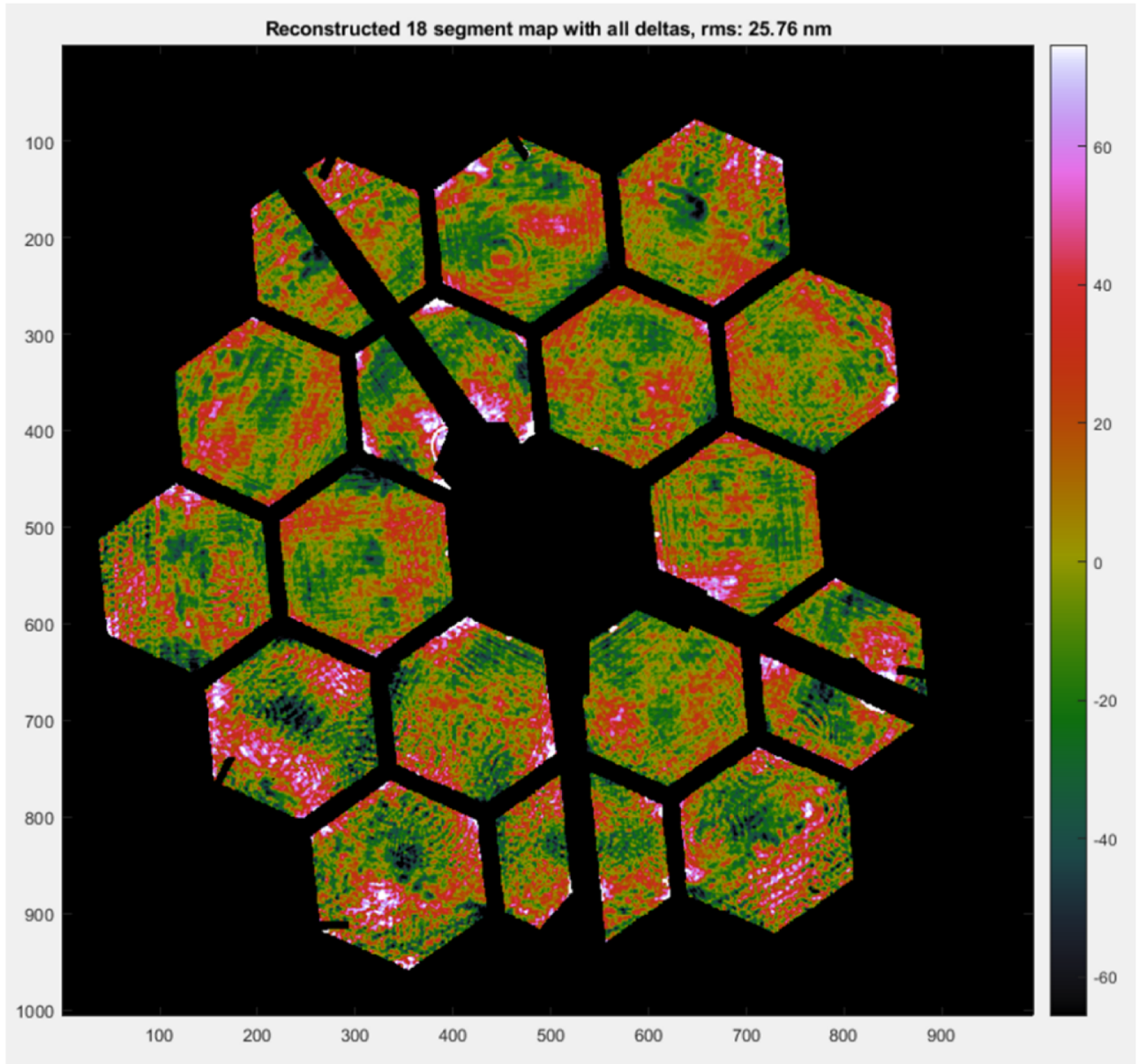

Result of $25.8 \mathrm{~nm}$-rms within estimated prediction/measurement/registration uncertainty of $26 \mathrm{~nm}$-rms. One PMSA not shown due to deformation discussed above. 

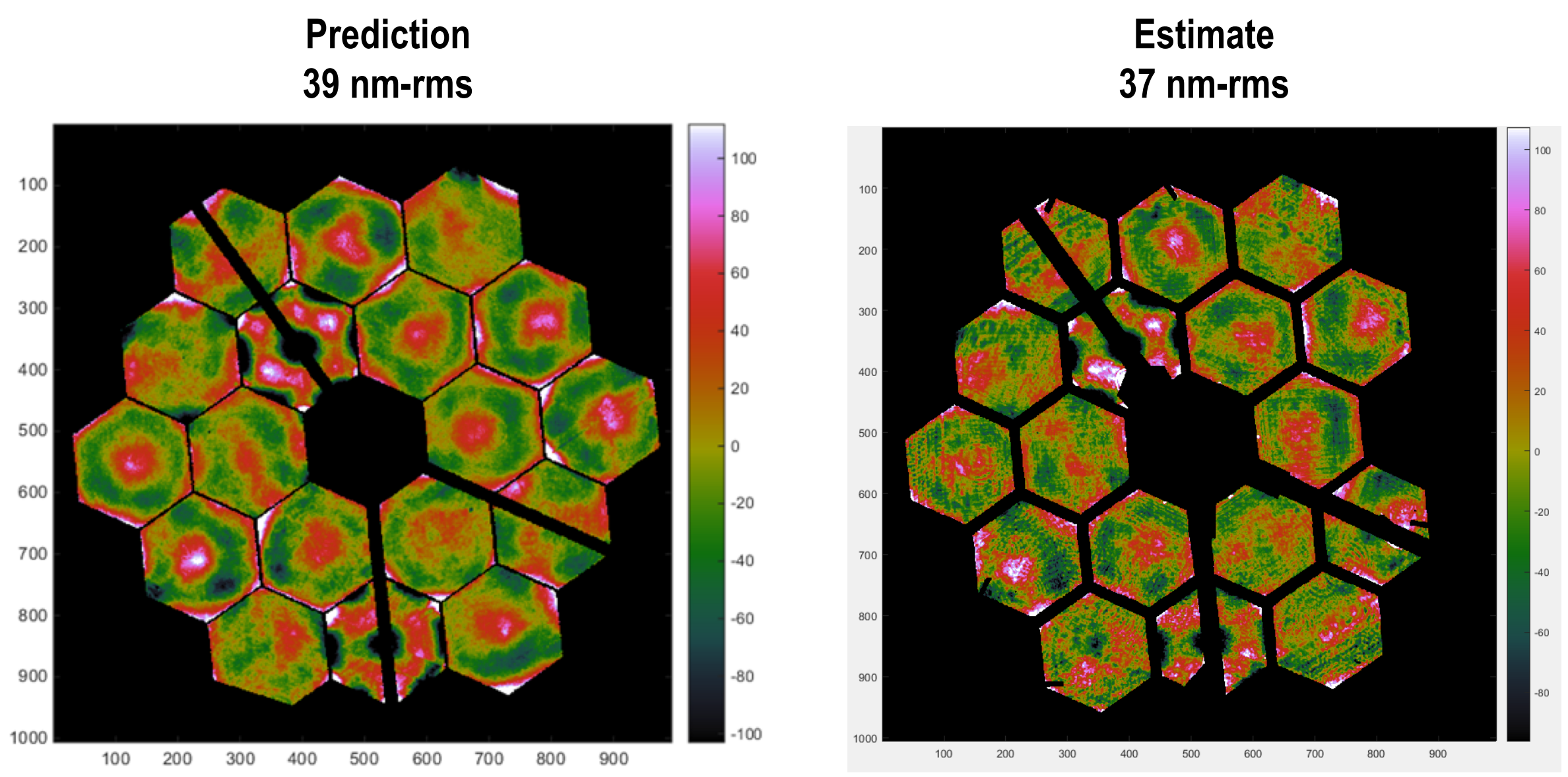

Prediction from $0 \mathrm{~g}$ WFE of each PMSA from cryogenic acceptance testing. Estimate from removal of $1 \mathrm{~g}$ deformations from JWST cryogenic test measurement. Excellent visual \& magnitude correlation. 


\section{Summary \& Conclusions}

- All test objectives accomplished.

- Aligned PMSAs into phased PM, with proper ROC \& conic constant.

- Measured phased PM WFE, ROC, conic constant, \& collecting area.

- Aligned PM globally to AOS.

- Aligned PM sufficiently for all other testing.

- All test requirements met.

- Measured 1g PM WFE matched prediction to within tolerance.

- Estimated Og PM WFE, with excellent correlation to prediction.

- Measured PM ROC \& conic constant within required uncertainties.

- Measured PM collecting area matched prediction to within tolerance. 